\title{
Suicidal ideation in seropositive patients seen at a South African HIV voluntary counselling and testing clinic
}

\author{
RD Govender ${ }^{1}$, L Schlebusch² \\ 1Department of Family Medicine, University of Kwa-Zulu Natal, Nelson R Mandela School of Medicine, Durban, South Africa \\ 2Department of Behavioural Medicine, University of KwaZulu-Natal, Nelson R Mandela School of Medicine, Durban, South Africa
}

\begin{abstract}
Objective: Suicidal behaviour and HIV/AIDS are significant public health concerns. The aim of this study was to investigate suicidal ideation in patients who were referred to a voluntary HIV counselling and testing (VCT) clinic and who were found to be seropositive. This in order to improve suicide prevention and intervention strategies amongst such patients. Method: The sample studied consisted of volunteer adult patients referred over a three-month period to a HIVVCT clinic based at a university-affiliated general state hospital. Patients completed a questionnaire on sociodemographic data. Suicidal ideation was measured using the Beck Hopelessness Scale and the Beck Depression Inventory (BDI), at two time points (within 72 hours after notification and again at a 6 week follow-up). All patients received extensive pre-and post-test counselling. Results: HIV-test results were available for 189 (99.5\%) of the original sample of 190 patients studied, with 157 (83.1\%) testing positive. More females tested positive as did unemployed and single/divorced patients. The mean age for HIV-positive patients was 33.49 (SD = 9.449), and for HIV-negative patients it was $37.94(\mathrm{SD}=15.238)$. Age was a significant factor in that for each year increase in age, the risk of testing HIV-positive decreased by $4.1 \%$. Lower education and traditional beliefs were also significantly associated with testing HIV-positive. At 72 hours suicidal ideation was present in $17.1 \%$ (95\% confidence interval $12.16 \%$ to $23.45 \%$ ), and at 6 weeks in $24.1 \%$ (95\% confidence interval $17.26 \%$ to $32.39 \%$ ) of the seropositive patients. Their average BDI scores were 15.20 and 14.23 respectively at the two time points. Conclusion: Suicidal ideation was present in a significant number of the seropositive cohort studied and increased over a six week period among these patients. The average BDI scores at both time points imply a clinical depression. The findings also suggest an association between positive HIV-test results and certain socio-demographic variables that can act as indicators for suicidal ideation in HIV-infected persons, although this requires further research. Although the relationship between suicidal ideation and HIV-infection is complex, it is an important consideration when assessing patient suicide vulnerability at HIV VCT clinics and when implementing suicide prevention and management strategies.
\end{abstract}

Keywords: Suicidal ideation; HIV-infection; Voluntary counselling and testing

Received: 10-12-2010

Accepted: 12-05-2011

doi: http://dx.doi.org/10.4314/ajpsy.v15i2.12

\section{Introduction}

Voluntary counselling and testing (VCT) offer patients an optional opportunity to be counselled and tested for the presence of HIV antibodies. Knowledge of their HIV status has major implications for HIV-positive persons. They include, inter alia, the need to disclose their status to their partner or partners, the need to change sexual behaviour,

Correspondence:

Professor L Schlebusch

Department of Behavioural Medicine, University of KwaZulu-Natal,

Nelson R Mandela School of Medicine, Durban, South Africa

email: schlebuschl@ukzn.ac.za accessing the availability of treatment and social support, as well as dealing with certain negative ramifications such as possible social stigmatization, employment uncertainty and adverse psycho-social-cultural factors. Additional considerations are: fear of adverse sequelae of the disease itself and psychiatric complications. The latter can include acute stress reactions, adjustment disorders, obsessive compulsive disorders involving obsessive ruminations and scrutiny for disease progression, bereavement reactions, neuropathology/cognitive impairment, personality disorders, depression, mania, psychoses, substance abuse, an exacerbation of pre-existing psychiatric disorders, neuropsychiatric side-effects that can result from ARV and 
psychotropic medication, anxiety reactions associated with the "worried well", "AIDS phobia", AIDS panic" or AIDS anxiety" and suicidal behavior. ${ }^{1-4}$

South Africa, a developing country, is considered to be one of the countries in the world worst affected by HIV/AIDS where about 5.7 million people and one in three pregnant woman are living with HIV/AIDS. ${ }^{1-2,5}$ The province of KwaZulu-Natal (where this study was done) has one of the highest seropositive prevalence rates. ${ }^{2}$ Various HIV prevention strategies have been launched in South Africa. Nearly $85 \%$ of all South African youth have been exposed to such national multi-media HIV education and awareness campaigns. ${ }^{6}$ However, these and other prevention campaigns have not diminished the progression of HIVinfection with heterosexual sexual intercourse being the main mode of HIV transmission. Infection rates remain high, especially amongst younger people with females more likely to be infected than males, ,7-13

Likewise, suicidal behaviour in South Africa is a major public health concern accounting respectively for an average non-natural death rate of $9.5 \%$ in young people and $11 \%$ in adults, giving overall suicide rates that range between 17.2 and 25 per 100000 of the population. ${ }^{2,14-16}$ It is estimated that for every suicide in the country there are at least 20 attempts ${ }^{2}$, and studies show that suicidal ideation is present in $12 \%$ amongst adolescent ${ }^{17}$ and $9.1 \%$ amongst adults ${ }^{18}$ in the general population. Further, an increased risk of suicidal behaviour in South Africa has been reported in HIV/AIDS sufferers. ${ }^{1,2,14}$ Nevertheless, it has been pointed out that there is a hidden burden of suicidal behaviour in the country that requires further research. ${ }^{1,2}$ For example, studies elsewhere ${ }^{4,19-20}$ have examined the mental health status of persons with acute/early HIV-infection as well as suicidal ideation associated with HIV testing, but there is a dearth of knowledge in this regard in developing countries such as South Africa despite its high suicide and HIV prevalence rates.

In light of the foregoing, the present study investigated suicidal ideation in patients referred to an HIV VCT clinic and who were found to be seropositive in order to improve suicide prevention and intervention strategies in these patients. Because depression is frequently associated with suicidal behaviour and ideation $2,4,16,19$ the presence of depressive symptomatology in the seropositive patients was also studied.

\section{Method}

\section{Participants/Setting}

The sample studied consisted of adult volunteer patients referred to an HIV VCT clinic at a university-affiliated general state hospital, Durban, South Africa over a three month period. The study was ethically approved by the Biomedical Research Ethics Committee of the University of KwaZulu-Natal. Patients voluntarily signed an informed consent form, and were given the right of choice not to answer any questions that they did not want to. All patients entered into the study received extensive pre- and post-test counselling tailored to the individual needs of each patient, risk factors, patient vulnerabilities, coping/supportive resources and knowledge about HIV transmission and its treatment.

\section{Measures}

Suicidal ideation was measured using the Beck Hopelessness Scale (BHS ) and the Beck Depression Inventory (BDI). The BHS is designed to measure hopelessness (negative expectations of the future) which can be used as an indirect indicator of suicide risk. ${ }^{21}$ It contains 20 true-false items and the severity of hopelessness is calculated by summing scores for the 20 items with the total score ranging from $0=$ no hopelessness to $20=$ maximum hopelessness. ${ }^{21}$ The BDI was developed to standardise the measurement of depression severity in order to monitor change over time..$^{21}$ It covers 21 behavioural manifestations (items) describing symptoms from low to high. Items are scored individually from 0 to 3 and then summed to obtain a total score ranging from 0-63 with $<9=$ minimal depression to $>30=$ severe depression. Both the BHS and the BDI were individually completed by each patient entered into the study at two time points, that is, within 72 hours after notification and again at a six week follow-up by arranging for patients to return to the VCT clinic. This time frame is

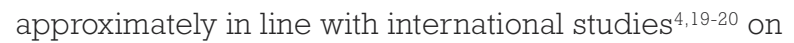
suicidal ideation and HIV-testing. On item 9 of the BDI (which was used to assess suicidal ideation) patients endorsed one of the following: 0 ("I don't have any thoughts of killing myself"), 1 ("I have thoughts of killing myself, but I would not carry them out"), 2 ("I would like to kill myself"), or 3 ("I would kill myself if I had the chance"). As in other research for the purpose of the present study, "suicidal ideation" was defined as a score of 1 to 3 on this item of the BDI. ${ }^{19}$ In addition, on the BHS and BDI total summed cut off scores respectively of 9 and 10 were used to determine suicidal ideation and depression. ${ }^{21}$ The patients also completed a questionnaire on sociodemographic variables grouped into seven correlates (categories), viz.: age, gender, educational status, employment, marital status, ethnicity and traditional beliefs To accommodate the isiZulu speaking patients in the sample (who constituted the largest African ethnic group), the BHS, BDI and socio-demographic questionnaire were translated into isizulu and then back translated with the assistance of a professional translator.

\section{Statistical Analysis of Data}

The SPSS version 15.0 (SPSS Inc, Chicago, Illinois, USA) was used for analysis of data. Pearson's Chi-square $\left(\mathrm{x}^{2}\right)$ test, t-tests and a binary logistic regression analysis of the socio-demographic variables were done. The latter used a backward stepwise method with entry and exit probabilities set at 0.05 and 0.1 based on likelihood ratio tests. The socio-demographic variables were entered into the model at step one. After five steps, the final model was reported with odds ratios and 95\% confidence intervals.

\section{Results}

HIV-test results were available for 189 (99.5\%) of the referred number of 190 patients. In total 83.1\% ( $\mathrm{n}=157)$ tested positive for HIV infection. A substantial portion of the patients studied had suicidal ideation at both time points which increased by $7 \%$ from the first to the second measurement (i.e. between 72 hours and the 6-week 
follow-up). This consisted of endorsement about thoughts of killing themselves on item 9 of the BDI and an average BHS cut-off score of 9 or above. Their average BDI scores, indicating clinical depression, also remained elevated at both time points.

At 72 hours following HIV testing, suicidal ideation was present in $17.1 \%$ of the seropositive sample (95\% confidence interval $12.16 \%$ to $23.45 \%$ ). The average BDI score was 15.20 (SD=5.917). At 6 weeks following HIV testing, suicidal ideation was found in 32 of 133 patients who had tested positive, giving a $24.1 \%$ risk (95\% confidence interval $17.26 \%$ to $32.39 \%$ ). The average BDI score for these patients was 14.23 (SD=5.849).

Suicidal ideation was not separately analysed for each socio-demographic correlate studied, but as a secondary consideration, collectively they suggest aetiological implications for HIV-positivity and suicidal ideation. Some patients were lost on follow-up and not all respondents provided information on all the variables investigated. The sub-totals analysed, therefore, vary as indicated in the text.

The mean age for the seropositive patients was 33.49 years ( $\mathrm{SD}=9.449$ ), and for the seronegative patients it was 37.94 years $(\mathrm{SD}=.15 .238)$. Ages ranged from 16 years to 79 years, the statistical significance of which is reflected in Table I. There was a borderline statistically significant association between gender and HIV status (Pearson's Chi Square 3.89, $\mathrm{p}=0.049)$, in that females $(85.6 \%)$ were more likely to test positive than males (73.1\%).

A significant association was found between education levels and HIV-test results (Pearson's Chi Square =18.5, $\mathrm{p}=0.001$ ). As education levels increased, the number of patients who tested positive for HIV infection decreased. As opposed to only $46.7 \%$ who tested positive in those with a tertiary education, (95.1\%) tested positive in those with no formal or only a primary education. Compared to the other educational levels, $81.6 \%$ with a Grade $8,84.6 \%$ with a Grade 10 and 83.7\% with a Grade 12 educational level, respectively were HIV-positive.

Of the unemployed, $88.3 \%$ tested positive, compared to students (75.0\%) and employed (75.8\%) patients. However, this difference was not quite statistically significant (Pearson's Chi square $=5.2, \mathrm{p}=0.074$ ). The single/divorced group was most likely to test HIV positive (85\%) compared to married adults (72.4\%), but this difference was not statistically significant (Pearson's Chi square 2.7, p=0.096). There was a significant association between ethnicity and
HIV-test results (Pearson's Chi square 21.2, p<0.001). The African ethnic group was significantly more likely to test HIV positive than the other ethnic groups in the sample studied (86\% vs 30\%).

An association was also found between traditional beliefs and HIV-test results (Pearson's Chi square 25.8, $\mathrm{p}<0.001$ ). Those who adhered to traditional African beliefs were significantly more likely to test HIV-positive than the non-traditional belief group (87.3\% vs 37.5\%).

The binary logistic regression analysis is shown in Table I. A significant association was found between the HIV-test results and age on the logistic regression $(\mathrm{OR}=$ $0.959, \mathrm{p}=.060$ ). With every one year increase in age, the risk of being positive decreased by 0.041 (4.1\%). The highest rate of HIV-seroconversion for both men and women in this study was between the ages of 26 to 34 years. When all factors were accounted for, a higher age became a protective factor against HIV-infection. Education was significantly associated with testing HIV-positive. Having no education or only primary education resulted in an almost 20 times higher risk for testing HIV-positive compared to those with a tertiary education. Similarly those with only grade 8, 10 or 12 education were also significantly more at risk than those with a tertiary education. Traditional beliefs also indicated a 22.6 times higher risk for testing HIV-positive compared to those who subscribed to non- traditional beliefs.

\section{Discussion}

Suicidal behaviour is a complex phenomenon, and risk factors and aetiology are multifactorial and multidimensional. However, in the present study, results corroborate both South African ${ }^{1-2,16}$ and international research ${ }^{19-20}$ findings that HIV/AIDS can be a risk factor in suicidal behaviour, and that in some patients suicidal ideation can be associated with HIV testing and notification. The findings also suggest an association between positive HIV-test results and certain sociodemographic variables that can act as indicators for suicidal ideation in HIV-infected persons. The high seropositive rate was expected in view of the facts that patients were referred and that the area serviced by the VCT clinic has one of the highest HIV-infection rates in the country. The ethnic distribution in the study was to be expected, as it reflects the demographics of the population served by the VCT clinic where the research was done.

\section{Table I: Binary logistic regression}

\begin{tabular}{|c|c|c|c|c|c|c|}
\hline & \multirow[t]{2}{*}{ Wald } & \multirow[t]{2}{*}{$d f$} & \multirow[t]{2}{*}{ p value } & \multirow[t]{2}{*}{$O R$} & \multicolumn{2}{|c|}{$95.0 \%$ C.I. for OR } \\
\hline & & & & & Lower & Upper \\
\hline Age (Years) & 3.528 & 1 & 0.060 & .959 & .918 & 1.002 \\
\hline Education & 10.284 & 4 & 0.036 & & & \\
\hline None vs tertiary & 9.293 & 1 & 0.002 & 19.694 & 2.898 & 133.818 \\
\hline Grade 8 vs tertiary & 4.715 & 1 & 0.030 & 5.545 & 1.182 & 26.020 \\
\hline Grade 10 vs tertiary & 5.205 & 1 & 0.023 & 5.406 & 1.268 & 23.043 \\
\hline Grade 12 vs tertiary & 4.425 & 1 & 0.035 & 4.738 & 1.112 & 20.188 \\
\hline Traditional African beliefs vs other beliefs & 13.355 & 1 & $<0.001$ & 22.630 & 4.247 & 120.571 \\
\hline Constant & 1.453 & 1 & 0.228 & .227 & & \\
\hline
\end{tabular}


The fact that there were more females than males and younger patients with suicidal ideation who tested positive for HIV-infection is supported by other research that shows women's and the youth's vulnerability in this regard. ${ }^{1-2,14,16}$ The age of sexual debut, especially in the young has been linked to having a greater number of sexual partners and therefore a greater risk of HIV infection. ${ }^{7-9}$ The effects of age mixing differ for males and females with young females who have older male partners also at a greater risk for HIV infection. ${ }^{8}$ These are important considerations given our findings, since in South Africa, as in many other countries, suicidal behaviour in young people is an increasing problem. , $^{2,15}$ This shift in relation to age is sometimes referred to as the "ungreying phenomenon." "2 In addition, consistent with international research, South African studies generally report a female preponderance in non-fatal suicidal behaviour in the younger age groups. ${ }^{2,14-15}$

It is not surprising, that increasing age and higher education in this study were found to be protective factors against contracting HIV- infection and associated suicidal ideation, while lower education and traditional beliefs did not appear to offer the same protection. Educational attainment and the relationship between HIV-infection is closely correlated with income, gender, employment/ socioeconomic status, economic migration and urbanisation with related socio-economic implications. ${ }^{11-}$ 12,23-24 Although some studies on the relationship between socio-economic changes and suicidal behaviour have produced divergent results, others have highlighted the impact on suicidal behaviour of both adverse socioeconomic factors as well as economic development. 2,15-16 Contemporary research ${ }^{25}$ has shown that low socioeconomic status increase the risk of suicidal behaviour, as does low educational levels and long term unemployment. According to South African research ${ }^{2,15}$ the role of socioeconomic correlates that contribute to suicidal behaviour risks in particular are: financial problems and associated feelings of loss of parental support, the effects of rapid urbanization, an increasing competitiveness in education and employment as well as rising expectations in the young as they move away from traditional value systems and norms. Regarding the role of traditional beliefs found in the present study, both international and African research ${ }^{16}$ has documented the potential influence of religious and cultural beliefs on suicidal behaviour.

The rates of suicidal ideation following HIV-testing in this study are comparable to those reported by other research, although on follow-up assessments the increases tend to vary. ${ }^{4,19}$ An important finding in the present research was that, despite extensive counseling, suicidal ideation increased from 17.1\% in the patients who initially underwent voluntary HIV-testing to nearly one quarter six weeks later in those who tested positive. This change in the suicidal ideation rate is almost twice the rates reported for the general population in South Africa. ${ }^{17-18}$ Finally, the average elevated BDI score of patients at both time points imply the presence of a clinical depression in the sample studied, which is consistent with the established link between suicidal ideation and depression. . $^{2,16,25}$

\section{Limitations}

Limitations of this study include the facts that the target population was urban based and that limited information on pre-existing psychiatric disorders, sexual history, and traditional and cultural beliefs restricted more in-depth exploratory analyses. Also, how the socio-demographic variables independently contribute aetiologically to suicidal ideation associated with seropositive HIV-test results requires further research. Although this study provided insight into suicidal ideation and seropositive results following HIV testing and notification, the results need to be interpreted with caution and cannot be axiomatically generalised.

\section{Conclusion}

Suicidal ideation which increased over a six week follow-up period was present in a significant number of the patients studied, and considerably higher than found in the general population reported in recent South African studies. ${ }^{17-18}$ Most suicidal acts are preceded by a process and suicidal ideation forms part of that process. ${ }^{2,25}$ However, as part of this process the dynamics are highly individual and the relationship between suicidal ideation, HIV-infection and the various socio-demographic factors found in this study are complex and interlinked. Nevertheless, it is hoped that the findings reported here will assist in assessing patient suicidal vulnerability at HIV VCT clinics when doing preand post-counselling and in implementing suicidal prevention and management strategies, especially given that suicidal ideation in these patients and their propensity for suicidal behaviour can be acute, chronic or latent and progress through various phases. ${ }^{1}$ As has been recommended before ${ }^{1}$, general suicide prevention guidelines s,25-27 $^{2}$ can be adapted to be socio-culturally sensitive for use in HIV-positive patients.

\section{Acknowledgements}

The authors wish to thank Prenisha Pillay for her research assistance, and Mrs. T Esterhuizen (Biostatistician) for statistical assistance. Dr RD Govender was supported by the Columbia University- South African Fogarty AIDS International Training and Research Programme (AITRP) funded by the Fogarty International Center, National Institute of Health (Grant\# D43TW00231).

\section{References}

1. Schlebusch L, Vawda N. HIV-infection as a self-reported risk factor for attempted suicide in South Africa. Afr J Psychiat 2010; 13: 280-283.

2. Schlebusch L. Suicidal Behaviour in South Africa. Pietermaritzburg. University of KwaZulu-Natal Press; 2005.

3. Lishman WA. Organic Psychiatry. The Psychological Consequences of Cerebral Disorder. 3rd ed. Oxford: Blackwell; 2005.

4. Atkinson JH, Higgins JA, Vigil O, Dubrow R, Remien RH, Steward $W T$, et al. Psychiatric context of acutelearly HIV infection. The NIMH multisite Acute HIV infection study:IV. AIDS Behav 2009; 13(6):1061-1067.

5. UNAIDS. AIDS epidemic update: 2009. Available at: <http://www.unaids.org/en/MediaCentre/PressMaterials/ FactSheets. asp > [last accessed 06 December 2010]. 
6. LoveLife: South Africa's National HIV Prevention Programme for Youth. Available at: http://www.kff.org/about/lovelife.cfm [last accessed 04 June 2009].

7. MacPhail C, Campbell C. I think condoms are good but, aai, I hate those things:condom use among adolescents and young people in a Southern African township. Soc Sci Med 2001 52(11):1613-1627.

8. Pettifor AE, Rees HV, Steffenson A, et al. Young people's sexual health in South Africa: HIV prevalence and sexual behaviors from a nationally representative household survey. AIDS 2005; 19:1525-1534

9. Pettifor A, O'Brien K, MacPhail C, Miller W C, Rees H. Early coital debut and associated HIV risk factors among young women and men in South Africa. Inter Perspect Sex Reprod Health 2009; 35(2):82-99

10. Abdool-Karim QA, Abdool-Karim SS, Singh B, Short R, Ngxongo S. Seroprevalence of HIV infection in rural South Africa. Aids 1992; 6:1535-1539

11. Sociocultural aspects of HIVIAIDS. Available at: http:// www.Health24 - HIV-Aids, The South African culture.htm. (last accessed 18 September 2009).

12. Barnighausen T, Hosegood V, Timaeus IM, Newell M L. The socioeconomic determinants of HIV incidence: evidence from a longitudinal, population-based study in rural South Africa. AIDS 2007; 21 (7):S29-S38

13. Auvert B, Taljaard D, Lagarde E, Sobngwi-Tambekou J, Sitta R, Puren A. Randomized, controlled intervention trial of male circumcision for reduction of HIV infection risk: the ANRS 1265 Trial. PLoS Med 2005; Nov 2 (11) : e298.

14. Schlebusch L, Burrows S. Suicides attempts in Africa. In Wasserman D. Wasserman C, eds, The Oxford Textbook of Suicidology and Suicide Prevention. A global perspective. Oxford: Oxford University Press, 2009: 105-108.

15. Schlebusch L. Suicidal Behaviour in Young People. Crime, Violence and Injury in South Africa: Enabling Child Safety. Tygerberg: Medical Research Council-University of South Africa 2012 (in press)

16. Schlebusch L. An overview of suicidal behaviour in Africa. In:
Contemporary Psychiatry in Africa: A Review of Theory, Practice and Research. Nairobi: Acrodile Publishing, 2011, 375-396.

17. Mashego AB, Madu SN. Suicide-related behaviours among secondary school adolescents in the Welkom and Bethlehem areas of the Free State province (South Africa). South Afr J Psychol 2009; 39 (4) : 489-506.

18. Joe S, Stein D J, Seedat S, Herman A, Williams DR. Prevalence and correlates of non-fatal suicidal behaviour among South Africans. Brit J Psychiat 2008;192: 310-311

19. Perry S, Jacobsberg L, Fishman B. Suicidal ideation and HIV Testing. JAMA 1990; 263(5):679-682

20. Perry S, Fishman B, Jacobsberg L, Young J, Frances A. Effectiveness of psychoeducational interventions in reducing emotional distress after human immunodeficiency virus antibody testing. Arch Gen Psychiatry 1991; 48(2): 143-147

21. American Psychiatric Association (APA). Handbook of Psychiatric Measures. Washington, DC: American Psychiatric Association; 2000

22. Ackermann L, de Klerk GW. Social factors that make South African women vulnerable to HIV infection. Health Care for Women International 2002; 23:163-172

23. Hargreaves J R, Bonell C P, Boler T, Boccia D, Birdthistle I, Fletcher $A$, et al. Systematic review exploring time trends in the association between educational attainment and risk of HIV infection in subSaharan Africa. AIDS 2008, 22:403-414.

24. Fenton L. Preventing HIVIAIDS through poverty reduction: the only sustainable solution? Lancet 2004; 364:1186-1187.

25. Wasserman D. Wasserman C, eds. The Oxford textbook of suicidology and suicide prevention. A global perspective. Oxford Oxford University Press, 2009.

26. Burrows S, Schlebusch L. Suicide prevention in South Africa. In Wasserman D. Wasserman C, eds, The Oxford Textbook of Suicidology and Suicide Prevention. A Global Perspective. Oxford: Oxford University Press, 2009, 105-108.

27. Schlebusch L, Burrows S, Vawda, N. Suicide prevention and religious traditions on the African Continent. In Wasserman D. Wasserman C, eds, The Oxford Textbook of Suicidology and Suicide Prevention. A Global Perspective. Oxford: Oxford University Press, 2009, 63-69

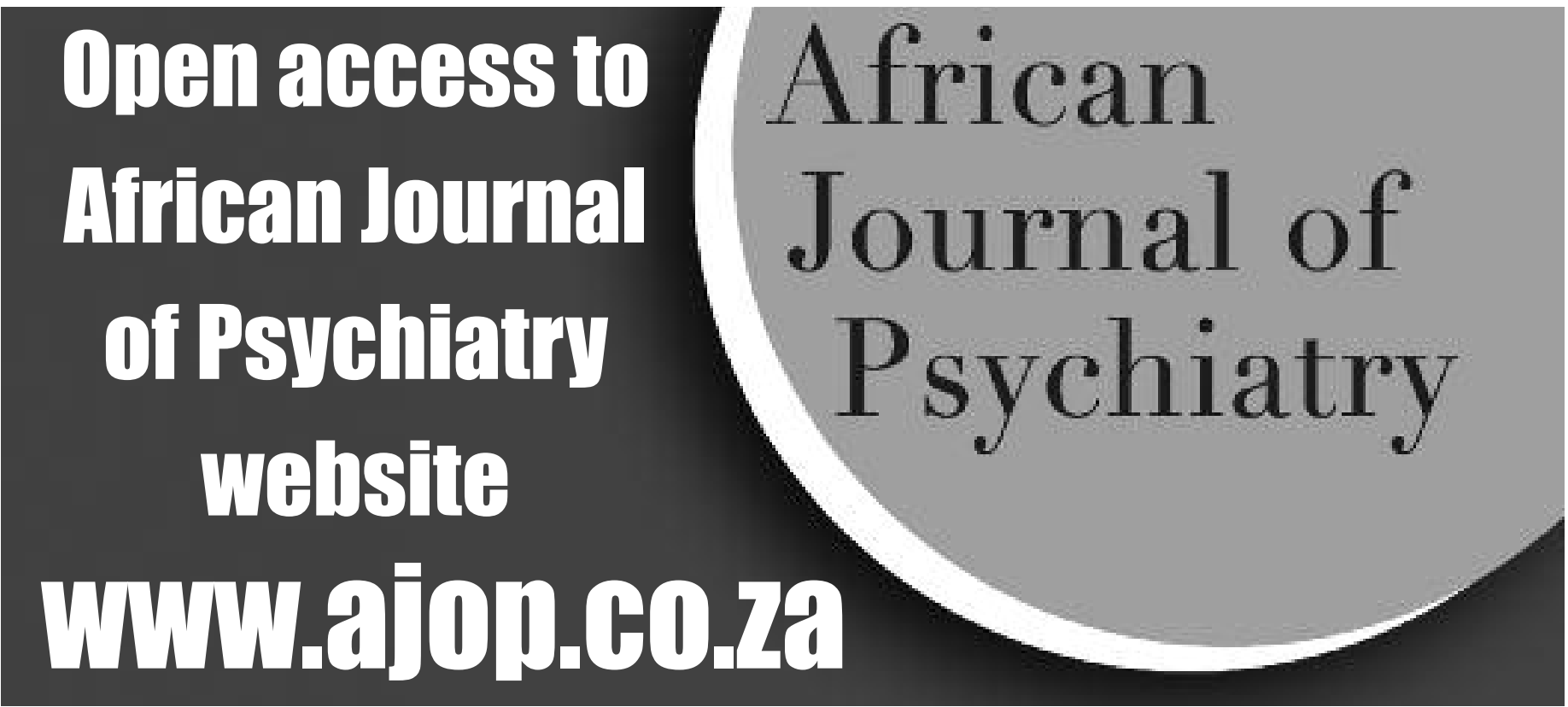

